\title{
HELICOPTER FUSELAGE DRAG - COMBINED COMPUTATIONAL FLUID DYNAMICS AND EXPERIMENTAL STUDIES
}

\author{
A. Batrakov ${ }^{1}$, A. Kusyumov ${ }^{1}$, S. Mikhailov ${ }^{1}$, V. Pakhov ${ }^{1}$, \\ A. Sungatullin ${ }^{1}$, M. Valeev ${ }^{1}$, V. Zherekhov ${ }^{1}$, \\ and G. Barakos ${ }^{2}$ \\ ${ }^{1}$ Kazan National Research Technical University \\ 10 K. Marx Str., Kazan 424011, Russia \\ ${ }^{2}$ School of Engineering, University of Liverpool \\ Liverpool L69 3GH, U.K.
}

\begin{abstract}
In this paper, wind tunnel experiments are combined with Computational Fluid Dynamics (CFD) aiming to analyze the aerodynamics of realistic fuselage configurations. A development model of the ANSAT aircraft and an early model of the AKTAI light helicopter were employed. Both models were tested at the subsonic wind tunnel of KNRTU-KAI for a range of Reynolds numbers and pitch and yaw angles. The force balance measurements were complemented by particle image velocimetry (PIV) investigations for the cases where the experimental force measurements showed substantial unsteadiness. The CFD results were found to be in fair agreement with the test data and revealed some flow separation at the rear of the fuselages. Once confidence on the CFD method was established, further modifications were introduced to the ANSAT-like fuselage model to demonstrate drag reduction via small shape changes.
\end{abstract}

\section{INTRODUCTION}

The use of CFD for the prediction of helicopter aerodynamics is a complex task because of the transient and three-dimensional (3D) nature of the flow around the main and tail rotors. Additional complexity arises due to the requirement to model the flow around the fuselage that in many cases behaves as a bluff body with flow separation at its rear part and around the engine exhausts, fuel tanks, skids, etc. The flow separation is partially responsible for an excess amount of drag on such bodies. Further, the inherent flow unsteadiness makes experimentation harder and calls for repeatable sets of measurements to result in data with

This is an Open Access article distributed under the terms of the Creative Commons Attribution License 4.0 , which permits unrestricted use, distribution, and reproduction in any medium, provided the original work is properly cited. 


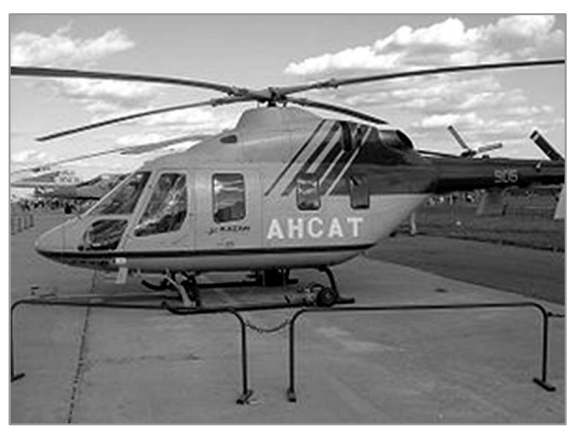

Figure 1 Photograph of ANSAT helicopter

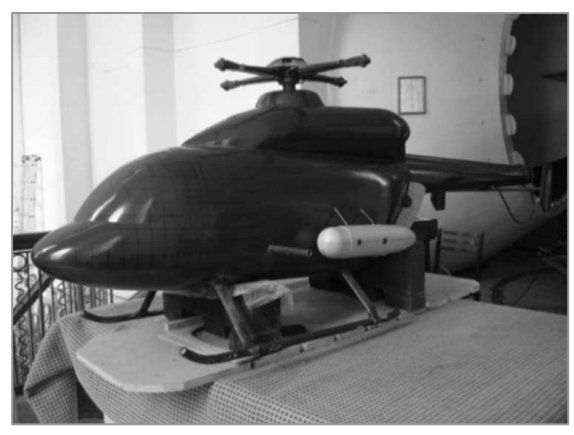

Figure 2 Wind tunnel model of the ANSAT-M2 helicopter fuselage

good confidence intervals. The focus of the present work is the CFD prediction of the helicopter fuselage aerodynamics. This topic has been the subject of several investigations and a good summary is provided in $[1-3]$.

A key aspect of the present work is the use of realistic fuselages with the main characteristics encountered in modern designs. For this reason, instead of idealized bodies, early development models of real helicopters were used. In order to have a fuselage representative of modern designs, an approximation to the ANSAT helicopter produced by the JSC Kazan Helicopters (Fig. 1) was first considered. A variant of this baseline fuselage referred to as ANSAT-M2 and shown in Fig. 2 was the first model considered. The ANSAT-M2 is one

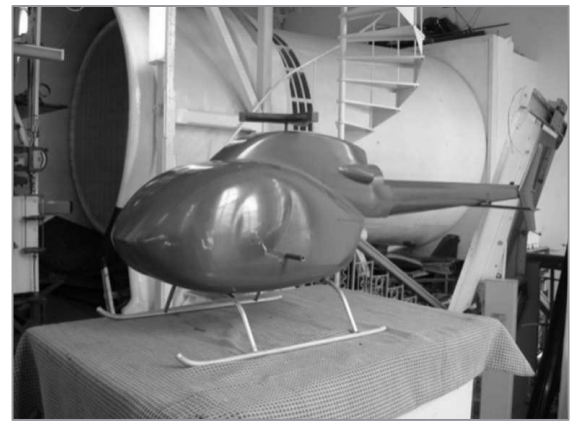

Figure 3 Wind tunnel model of the AKTAY helicopter fuselage of many initial designs employed during the development of the ANSAT aircraft and was used for preliminary aerodynamic studies conducted in the wind tunnel T-1K of KNRTU. The fuselage is not the exact shape of the ANSAT helicopter body with differences concentrated near the engine housing and exhausts.

The second case considered in this paper is an early model of the AKTAI light helicopter (Fig. 3). It is an asymmetric fuselage shape due to the requirements to house the engine and other equipment. The aerodynamics of several early models of the ANSAT helicopter has also been investigated and these results are presented in [4-7]. 
The numerical computations were performed using the in-house CFD tool HMB (helicopter multiblock), developed at Liverpool University. The CFD grids were constructed using the ANSYS ICEM commercial mesh generator. The computational domains were resolved using hexahedral grids and the $3 \mathrm{D}$ steady Reynolds-averaged Navier-Stokes (RANS) equations. The computation of the integral and distributed loads on an isolated helicopter fuselage was the first aim of the present investigation.

\section{COMPUTATIONAL FLUID DYNAMICS MESH GENERATION}

For the CFD grids around the ANSAT-M2 and AKTAY fuselages, the ICEM-Hexa tool has been used. The length of the ANSAT-M2 fuselage was $L_{F}=1.57 \mathrm{~m}$, and a reference area of $S_{F}=0.0694 \mathrm{~m}^{2}$ was used for computing the aerodynamic coefficients of lift and drag. The computational domain was divided in 764 blocks. For the AKTAY wind tunnel model, the geometric parameters were $L_{F}=1.64 \mathrm{~m}$ and $S_{F}=0.1075 \mathrm{~m}^{2}$, while the CFD grid required 1342 blocks. The grid was refined near the fuselage surface (and the resulting $y^{+}$values were near 1) to resolve the laminar sublayer for better predictions of the friction drag coefficients.

Figure $4 a$ presents a part of the multiblock topology of the grid as well as the surface mesh on the ANSAT-2M fuselage. The surface grid for the AKTAY fuselage is shown in Fig. 4b. A mesh convergence study was first conducted with respect to the number of surface cells and their spatial distribution.

For all fuselages, the results presented in this work are nearly mesh independent for the employed Re and turbulence model.

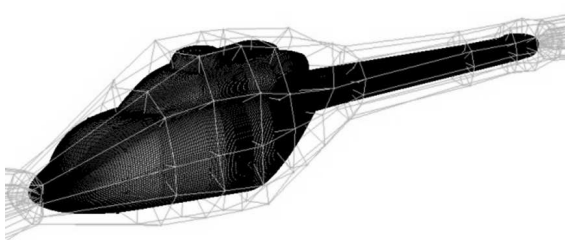

(a)

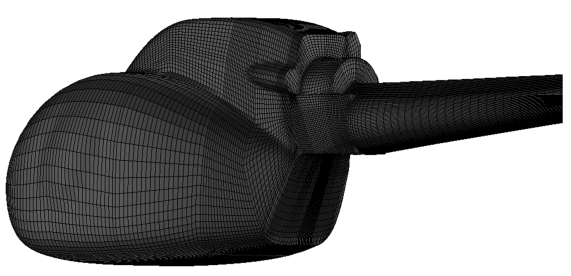

(b)

Figure 4 Surface mesh and multiblock topology for the ANSAT-2M fuselage $(a)$ and surface mesh on the AKTAY model $(b)$ 


\section{PREDICTION OF AERODYNAMIC FORCE COEFFICIENTS}

The open test section (of a 2.25-meter diameter) closed circuit, low speed, wind tunnel T-1K of KNRTU-KAI is equipped with a six-component Prandtl balance. Eight-times measurements were conducted to reduce random experimental errors (system errors, mounting of model, model manufacturing errors, etc.) and for plotting error bars around the obtained results.

The standard $k-\omega$ turbulence model was used for computations due to its popularity within the CFD $[8,9]$. The CFD validation was conducted at Reynolds number of $\operatorname{Re}=3,2 \cdot 10^{6}$ and at a free-stream Mach number of $\mathrm{M}_{\infty}=0.1$. These conditions correspond to the experimental investigations. Figure $5 a$ presents the CFD predictions of the lift and drag coefficients for the ANSAT-M2 model in comparison with the wind tunnel experiment data. The error bars shown on the graphs correspond to the experimental confidence intervals. Figure $5 b$ presents the corresponding $C_{D}$ for the AKTAY model. Figures $5 a$ and $5 b$ suggest
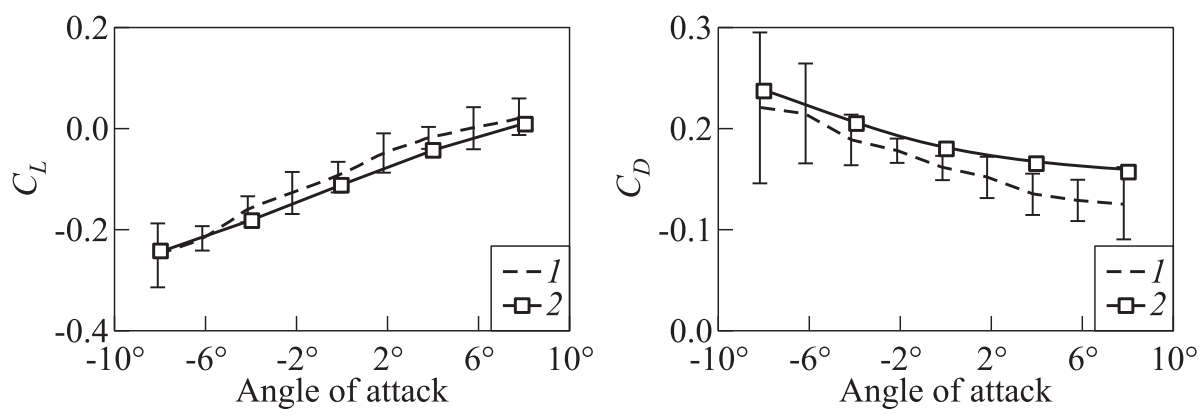

(a)
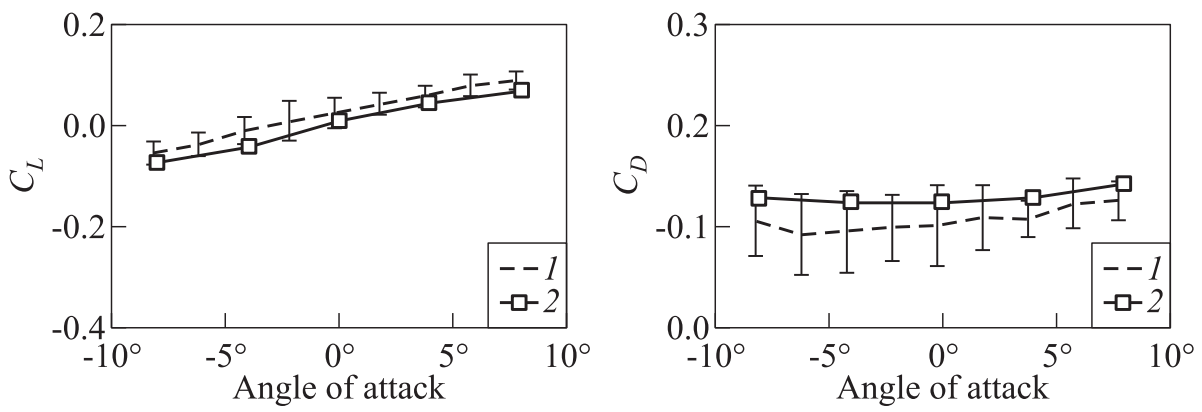

(b)

Figure 5 Experimental (1) and CFD (2) lift and drag coefficients vs. pitch angle for the ANSAT-M2 $(a)$ and AKTAY $(b)$ models 
a good agreement between $\mathrm{CFD}$ and experimental results for the lift and drag coefficients in the considered range of pitch angles. For all models, the drag coefficient values are overpredicted in comparison with the experimental data unlike the underpredicted lift coefficient data. The level of agreement with the experiments is satisfactory for a simple RANS model used for computations.

\section{DISTRIBUTED FLOW PARAMETERS}

The integrated loads are complemented by comparisons of distributed flow parameters that allow for a better understanding of the influence of the fuselage geometry on the flow structure. For postprocessing of the CFD results, the TECPLOT360 commercial visualization tool was used.

\subsection{Experimental and Computational Fluid Dynamics Predicted Vector Fields Comparison}

Figures $6 a$ and $6 b$ present a comparison of experimental and CFD-predicted vector fields for ANSAT-M2 and AKTAY models.

The experimental vector field was obtained using a two-dimensional (2D) PIV system. Figure 6 suggests a good qualitative agreement between CFD and experiments for the considered range of pitch angles. Both CFD and wind tunnel tests revealed a flow separation area although the extend of separation is different between the two models.

\subsection{Velocity Fields}

Figure 7 presents velocity distributions around the mid-plane of the fuselage, and shows the evolution of the separated flow regions with the pitch angle.

For the ANSAT-M2 model, increasing the pitch angle leads to growth of the separation area at rear part of fuselage, but it does not cause the same growth in the drag force (see also Fig. $5 a$ ). Moreover, according to the CFD and experimental data, the drag coefficient is monotonically decreasing with the pitch angle. For the AKTAY fuselage, the drag coefficient has approximately constant values within the considered range of pitch angles. Figure 8 presents the velocity fields at a horizontal plane under the tail boom of the ANSAT-M2 and AKTAY fuselages.

At $\alpha=8^{\circ}$, a separation area centered in the considered section is located at the symmetry plane of ANSAT-M2 fuselage (see Fig. $8 b$ ). For $\alpha=-8^{\circ}$, Fig. $8 a$ reveals the presence of two separation cores near the horizontal plane. 


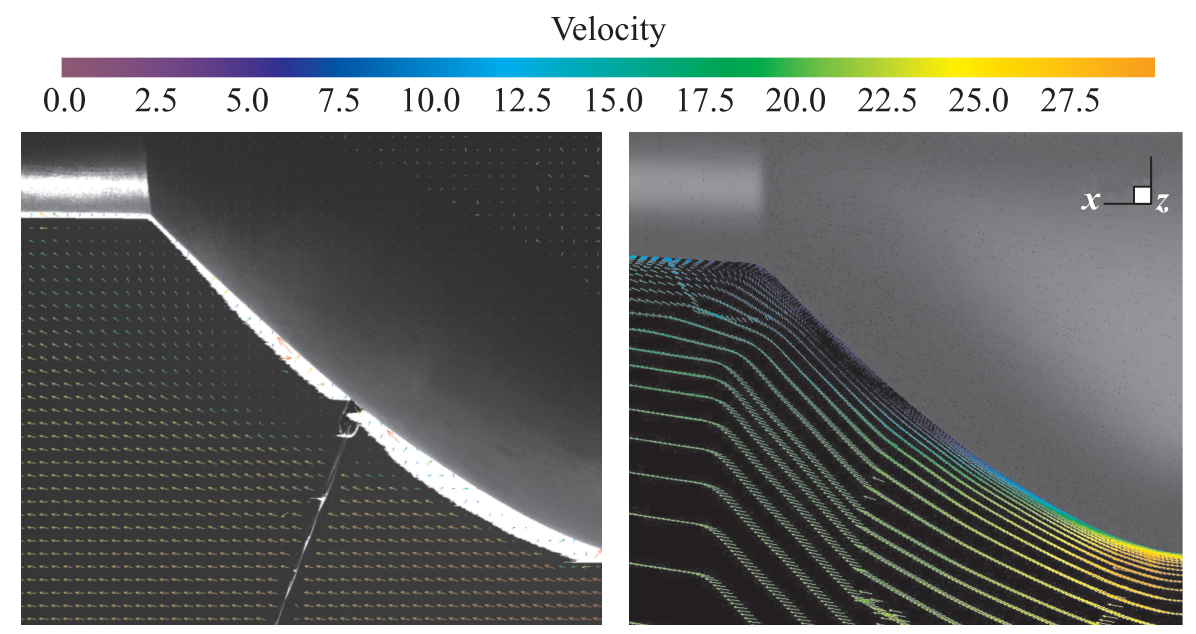

(a)

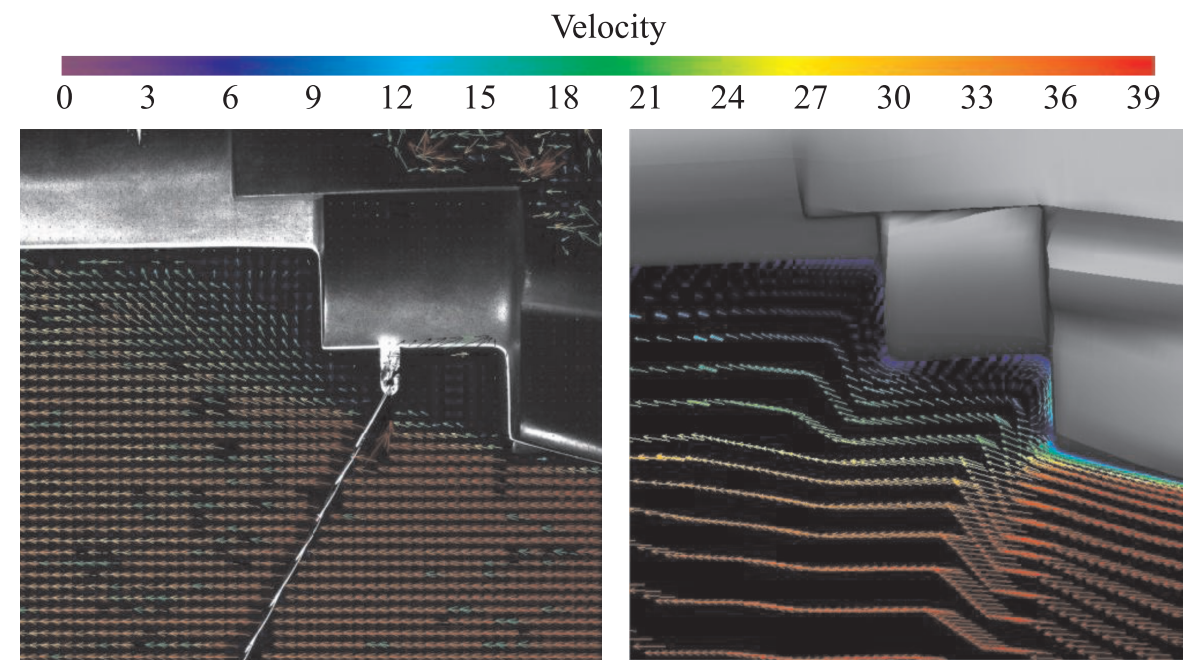

(b)

Figure 6 The PIV (left column) and CFD (right column) velocity vector fields at the mid plane of the ANSAT-M2 $(a)$ and AKTAY $(b)$ fuselages for $\alpha=0^{\circ}$. Comparison around the rear fuselage part is shown

The ANSAT-M2 geometry is more streamlined in comparison to the AKTAY model. For the AKTAY model, the topology of the separated area is the same for $\alpha=-8^{\circ}$ and $8^{\circ}$. For this reason, the drag coefficient value for $\alpha=-8^{\circ}$ is close to the drag coefficient for $\alpha=8^{\circ}$. This behavior was expected for a body 

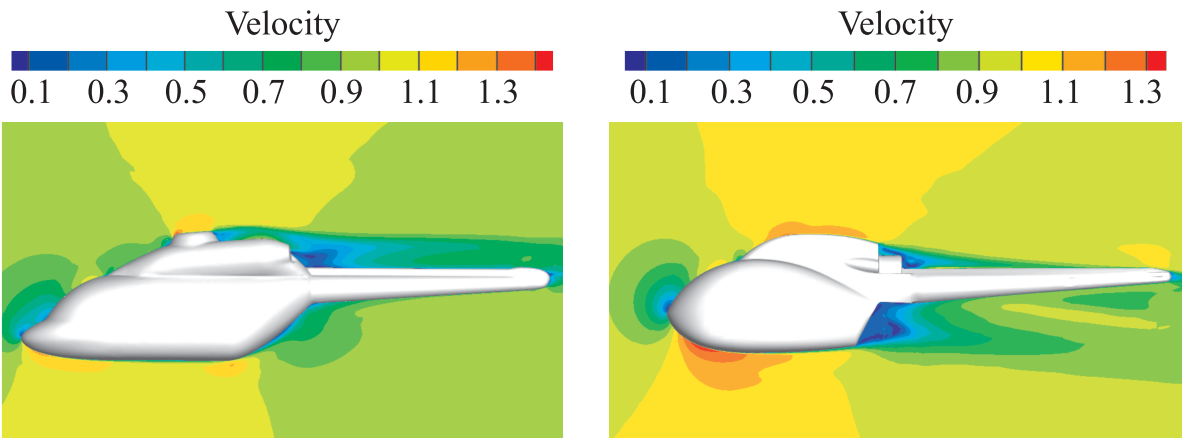

(a)
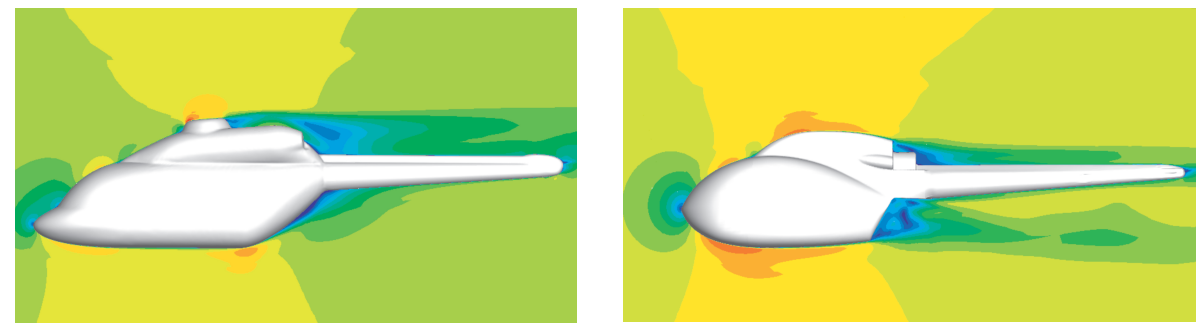

(b)
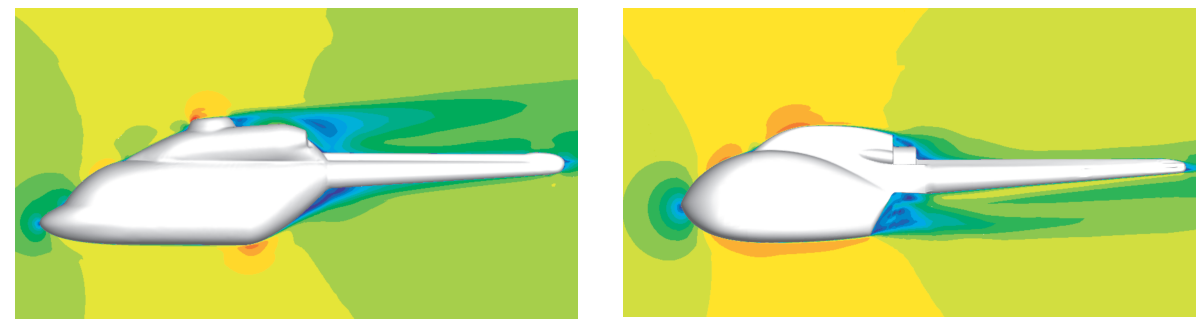

(c)

Figure 7 The CFD predictions of mid-plane velocity for the ANSAT-M2 (left column) and AKTAY (right column) fuselages at $\alpha=-8^{\circ}(a), 0^{\circ}(b)$, and $8^{\circ}(c)$

shaped like the AKTAY that has always separated the flow near the fuselage junction with the tail-boom.

\subsection{Further Flow Visualization}

Isosurfaces corresponding to velocity magnitude of $V=0.2 V_{\infty}$ are used for visualization where $V_{\infty}$ is the free-stream velocity. For areas without flow separation, 

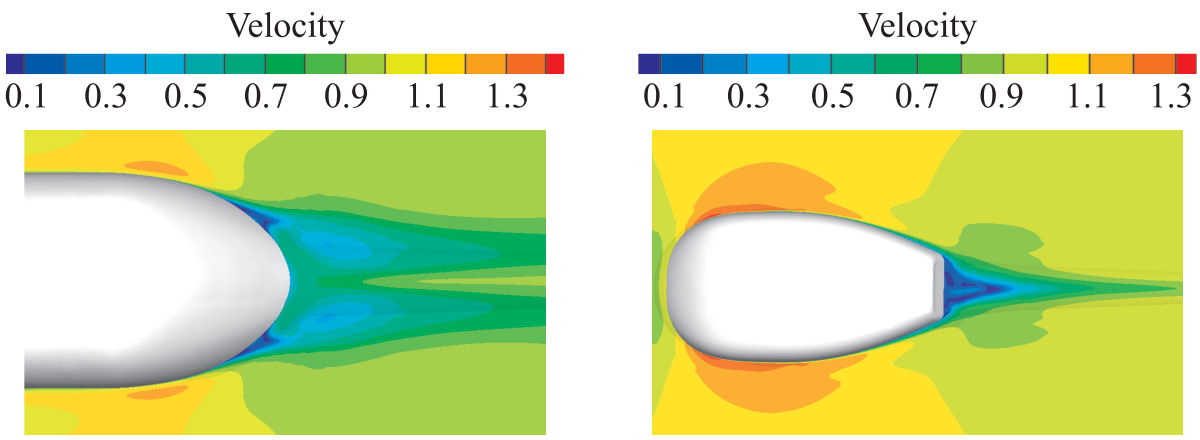

(a)
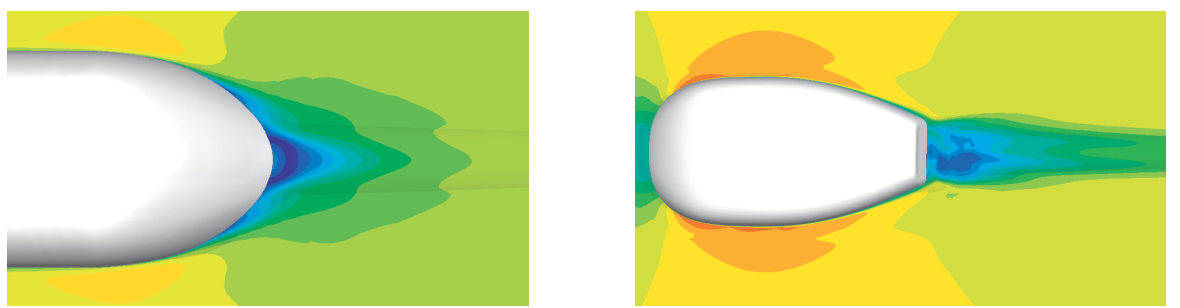

(b)

Figure 8 The CFD prediction of horizontal plane velocity for the ANSAT-M2 (left column) and AKTAY (right column) fuselages at $\alpha=-8^{\circ}(a)$ and $8^{\circ}(b)$

the isosurfaces of $V=$ const are close to the fuselage surface and the geometry of the isosurfaces corresponds to the fuselage geometry. In the separation zones, the isosurfaces detach from the fuselage surface and this shows the flow separation zones. Figure 9 presents the isosurfaces for different pitch angles.

Figure 9 shows the different character of the separation areas for the streamlined (ANSAT-M2) and the bluff (AKTAY) fuselages. In general, the shape of the isosurfaces corresponds to the flow fields presented in Fig. 8.

For the ANSAT-M2 model, there are two areas of separation with different character of development with respect to the pitch angle. Both areas of separation are located symmetrically to the fuselage mid-plane. This separation region is maximized for negative pitch angles and it maintains the same structure with two contra-rotating vortices. The intensity of the separation is maximum for a negative pitch angle of $-8^{\circ}$ and monotonically decreases with respect to the pitch angle. A different separated flow region with a single core of separation is located at the back of the fuselage, near the tail boom junction. The intensity of this separated flow region is maximum for a positive pitch angle of $8^{\circ}$. At 

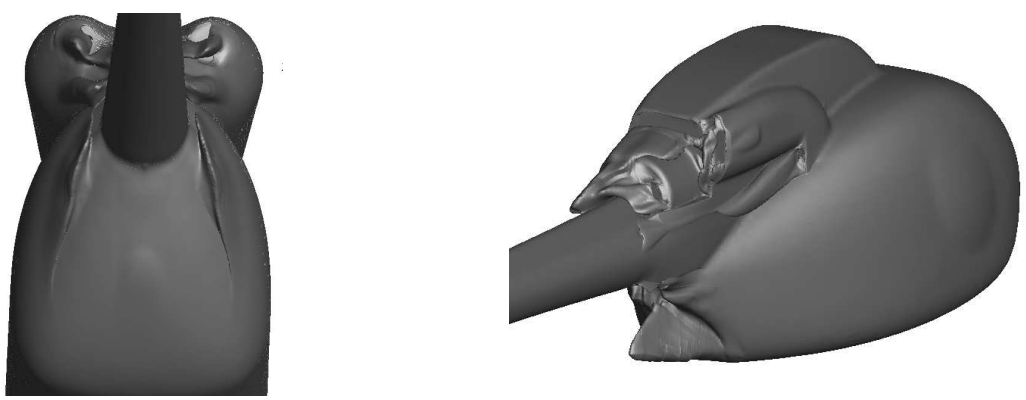

(a)
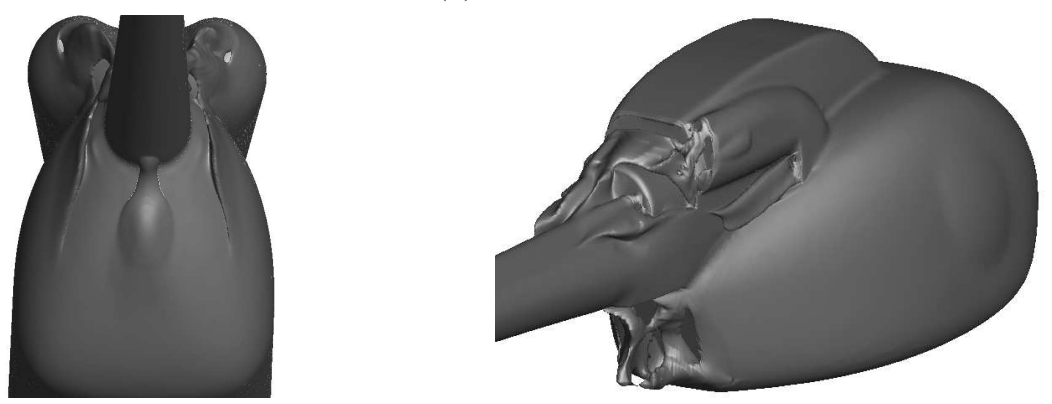

(b)
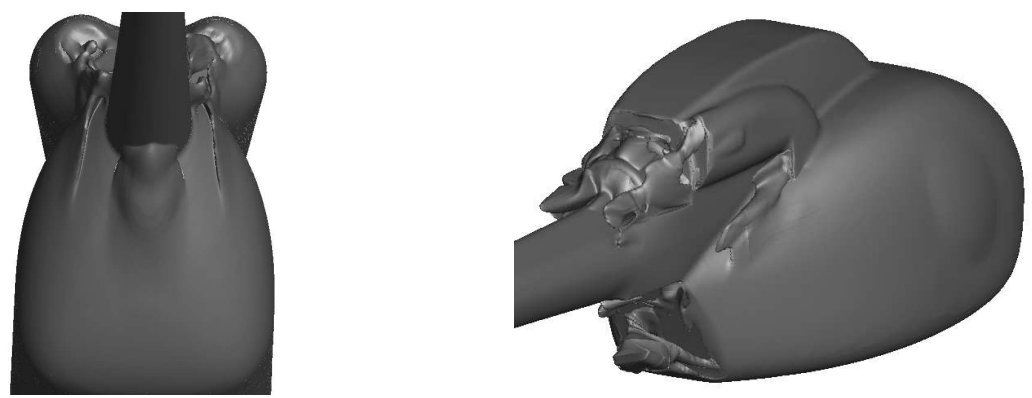

(c)

Figure 9 Isosurfaces corresponding to $V=0.2 V_{\infty}$ for the ANSAT-M2 (left column) and the AKTAY (right column) fuselages for $\alpha=-8^{\circ}(a) ; 0^{\circ}(b)$; and $8^{\circ}(c)$

a pitch of $-8^{\circ}$, this separation region almost disappears. The flow structure for the AKTAY model is different because of the bluff rear fuselage. For the AKTAY model, the flow at the rear part of fuselage has a more unsteady character and is less sensitive to the pitch angle. 


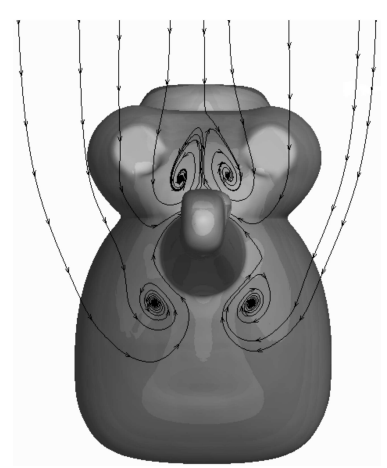

(a)
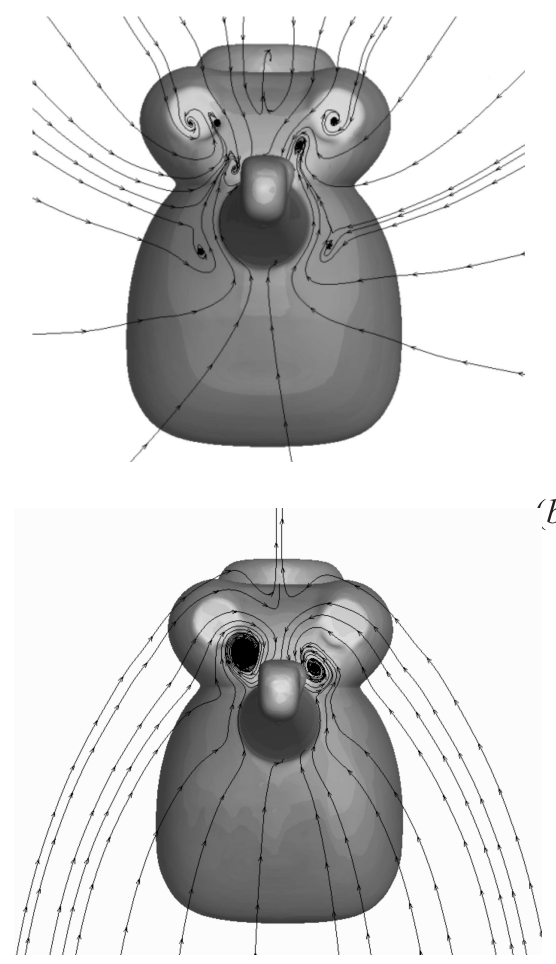
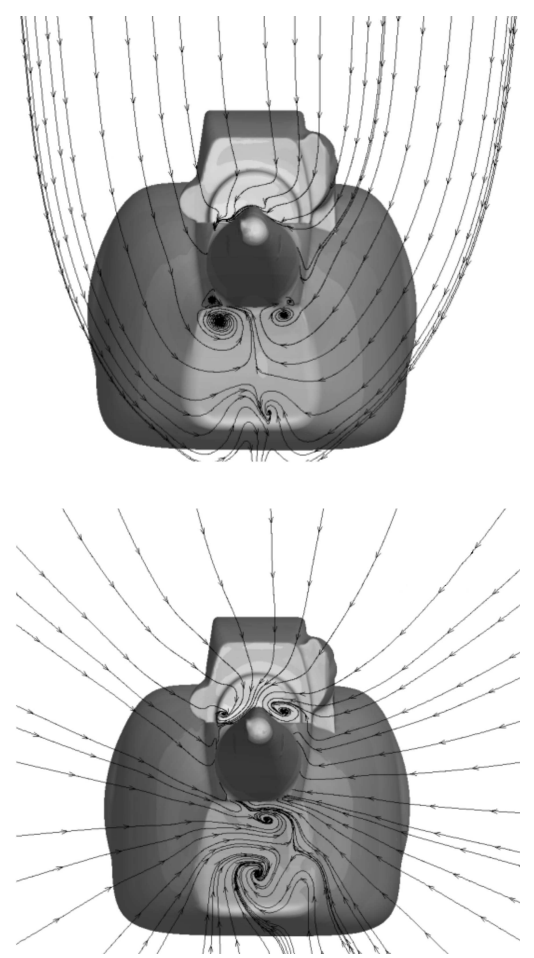

(b)

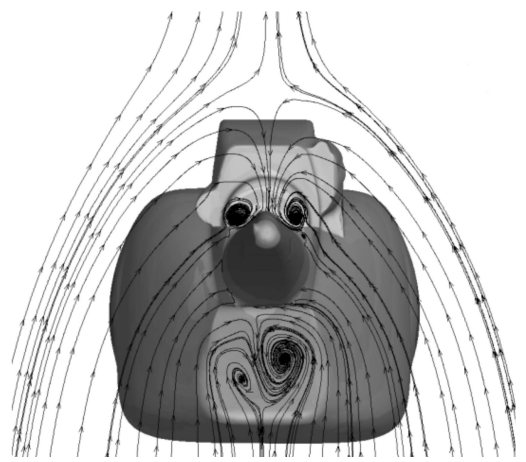

(c)

Figure 10 Stream functions for ANSAT-M2 (left column) and AKTAY (right column) fuselages for $\alpha=-8^{\circ}(a), 0^{\circ}(b)$, and $8^{\circ}(c)$ 


\subsection{Flow Stream Lines}

Figure 10 shows the stream lines on the $Z Y$ plane that is normal to the freestream velocity vector. For the ANSAT-M2 model, the location of vortices at the rear part of fuselage shows two cores of separation present at $-8^{\circ}$. At $0^{\circ}$ and $8^{\circ}$ of pitch, the flow is more attached. The flow structure at the rear part of AKTAY fuselage appears to be separated regardless of the pitch angle and the size of the separated flow region is independent of the pitch angle.

\section{CONCLUDING REMARKS AND FUTURE WORK}

The flows around the simplified ANSAT and AKTAY helicopter fuselages were analyzed. The experimental values of drag and lift coefficients were compared with CFD data with good agreement. The structure of the separated flow region at the rear of both fuselages was also analyzed at different pitch angles. It is shown that for the streamlined shape of the ANSAT-M2 model, the flow at rear part of fuselage has two main separation regions. This is not the case for the AKTAY where the flow separation covers a single large area, and is present regardless of the pitch angle. In the future, the effect of the helicopter rotor on the fuselage drag will be considered along with further investigations to design the rear fuselage for drag reduction in an optimal way.

\section{ACKNOWLEDGMENTS}

This work is supported by the "Leading Scientist" grant of the Russian Federation, under order 220 of the Russian Ministry of Education. The authors would like to acknowledge the Kazan Helicopter Plant for providing the initial fuselage shapes for this research.

\section{REFERENCES}

1. D'Alascio, A., K. Pahlke, and F. Le Chuiton. 2004. Application of a structured and an unstructured CFD method to the fuselage aerodynamics of the EC145 helicopter, prediction of the time averaged influence of the main rotor. ECCOMAS European Congress on Computational Methods in Applied Sciences and Engineering.

2. Le Chuiton, F., A. D'Alascio, G. N. Barakos, R. Steijl, D. Schwamborn, and H. Ludeke. 2009. EC145 helicopter fuselage - an industrial case. DESider A European effort on hybrid RANS-LES modelling. Eds. W. Haase, M. Braza, 
and A. Revell. Notes on numerical fluid mechanics and multidisciplinary design ser. Springer Verlag. 103:250-260.

3. Bridgeman, J. O., and G. T. Lancaster. 2010. Predicting hub drag on realistic geometries. American Helicopter Society Aeromechanics Specialists' Conference. San Francisco, CA, USA.

4. Kusyumov, A. N., S. A. Mikhailov, E. I. Nikolaev, N. A. Shilova, and A. O. Garipov. 2011. Simulation of flow around the fuselage of "ANSAT" helicopter. ASME 2011 International Mechanical Engineering Congress \& Exposition (IMECE2011). Denver, Colorado, USA.

5. Pahov, V., M. Valiev, V. Zherekhov, L. Makarova, A. Kusuymov, and G. Barakos. 2012. Creating a database for validation of predictive methods for rotorcraft. 47th Symposium (International) of Applied Aerodynamics. Paris, France.

6. Kusyumov, A. N., S. A. Mikhailov, E. V. Romanova, A. O. Garipov, E. I. Nikolaev, and G. Barakos. 2012. Simulation of flow around isolated helicopter fuselage. EFM 2012 Conference. Hradec Kralove, Czech Republic.

7. Kusyumov, A., S. Mikhailov, A. Garipov, E. Nikolaev, and G. Barakos. 2012. CFD simulation of fuselage aerodynamics of the "ANSAT" helicopter prototype. Trans. Control Mech. Syst. 1(7):318-324.

8. Lienard, C., A. Le Pape, and C. Verbeke. 2012. Numerical and experimental investigation of helicopter fuselage drag reduction using active flow control. American Helicopter Society 68th Annual Forum. Fort Worth, TX, USA.

9. Dietz, M., T. Kneisch, G. Roth, A. D'Alascio, and D. Schimke. 2012. EC145 T2: Comprehensive and challenging industrial CFD applications. American Helicopter Society 68th Annual Forum. Fort Worth, TX, USA. 